\title{
Application of the Mast resistotyping scheme to Campylobacter jejuni and C. coli
}

\author{
R. J. OWEN, E. LORENZ and J. GIBSON \\ Campylobacter Special Projects Unit, Laboratory of Enteric Pathogens, Central Public Health Laboratory, \\ London NW9 5HT
}

\begin{abstract}
The Mast resistotyping scheme was assessed with 228 strains of Campylobacter jejuni and $C$. coli from enteric infections in man and from a diverse selection of other sources (livestock, chickens and river water). Most (153 of 158) $C$. jejuni examined were of the three most common Penner (heat stable, HS) serotypes, HS1, HS2 and HS4 complex. Fourteen resistotypes were identified in the 158 strains of $C$. jejuni and 16 in the 70 isolates of $C$. coli. The predominant codes were $00(44 \%$ of $C$. jejuni; $33 \%$ of $C$. coli) and 40 (21\% of both species). The scheme was simple to use but reproducibility and interpretation of sensitivity zones - notably for fluorouracil, triphenyltetrazolium chloride and metronidazole - was occasionally problematic. Overall, resistotypes did not correlate with Penner HS serotypes or with three key genomic markers (ribotype, PFGE macrorestriction-type and $f(a$-type). Although resistotyping offers a rapid means for distinguishing between some strains of $C$. jejuni and $C$. coli, discrimination for common resistotypes can be achieved only in combination with other typing methods.
\end{abstract}

\section{Introduction}

Most human cases of campylobacteriosis are sporadic infections which are believed to be caused by Campylobacter jejuni and $C$. coli, although clinical laboratories in the UK rarely speciate isolates [1]. Surveillance data on foodborne infectious intestinal disease generally treat all such strains simply as Campylobacter spp. [2]. Outbreak investigations demand more detailed identification, and several biotyping schemes have been developed over the past 15 years as cost-effective and convenient phenotypic methods for characterising thermotolerant campylobacters.

The various biotyping schemes developed for C. jejuni also provide a basis for subtyping $C$. coli. Four biotypes of $C$. jejuni and two of $C$. coli were defined in the widely used Lior extended scheme [3] and greater discrimination was achieved in the Preston scheme $[4,5]$ in which three biochemical and 10 resistotyping tests were used. The advantage of that scheme was that it enabled most clinically important campylobacters to be speciated and biotyped. In the present study, the reproducibility and discriminatory

Received 25 April 1996; revised version accepted 5 June 1996.

Corresponding author: Dr R. J. Owen. power of the six-test Mast Campylobacter Biotyping Scheme developed as a simplification of the Preston scheme have been examined. Resistotypes were determined on a set of $C$. jejuni and $C$. coli isolates from human disease and from healthy animals. Discrimination was assessed by reference to Penner serotyping, and to genomic analyses based on previously published ribotyping and pulsed-field gel electrophoretic (PFGE) DNA macrorestriction profiles [6-8] together with fla-typing [9].

\section{Materials and methods \\ Bacterial strains and growth conditions}

The reference numbers and origins of the 158 strains of C. jejuni tested in this study were given previously with details on their Penner (HS) serotypes, 16S ribotypes and pulsed-field gel electrophoretic (PFGE) profiles [6-8]. Most strains were serotypes HS1 (52 strains), HS2 (36 strains) and HS4 complex (63 strains) and included 117 human isolates (41 HS1; 27 HS2; 44 HS4 complex; 2 HS 11; 1 HS44; 2 HS53), 21 bovine isolates ( 7 HS1; 7 HS2; 6 HS4 complex; 1 HS8/17), nine dog isolates (1 HS1; $8 \mathrm{HS} 4$ ), three chicken isolates (2 HS1; 1 HS12) and eight reference strains from the NCTC collection. Representative strains from two outbreak sets were examined and comprised five isolates (HS2) 
from a milk-borne outbreak in the Cardiff area [7] and four HS1 strains from a water-borne outbreak which occurred in the UK in 1988 [6]. The reference numbers and origins of the 70 strains of $C$. coli tested in this study were also given previously with details on their Penner serotypes, 16S ribotypes, flagellin gene ( $f a \mathrm{~A}$ ) types and PFGE profiles [8].

Bacterial isolates, mostly collected in the UK between 1993 and 1995, were preserved over liquid nitrogen on plastic beads in Nutrient Broth No. 2 (Oxoid CM67) $1 \% \mathrm{w} / \mathrm{v}$ containing glycerol $10 \% \mathrm{v} / \mathrm{v}$. Strains were subcultured initially on defibrinated horse blood agar $5 \% \mathrm{v} / \mathrm{v}$ for $24 \mathrm{~h}$ at $37^{\circ} \mathrm{C}$ under micro-aerobic conditions $\left(\mathrm{CO}_{2} 5 \%, \mathrm{O}_{2} 5 \%, \mathrm{H}_{2} 2 \%\right.$ and $\left.\mathrm{N}_{2} \quad 88 \%\right)$ in a Variable Atmosphere Incubator (Don Whitley Scientific Ltd, Shipley, Yorks).

\section{Resistotyping}

The resistotyping scheme consisted of the Mast Campylobacter (Camp Bioring) biotyping ring and
Campylobacter biotyping agar (a blood-free charcoal medium). The components were purchased from Mast Diagnostics (Bootle, Merseyside). The biorings comprised six arms with tips impregnated with: tetracycline (T $18 \mu \mathrm{g}$ ); nalidixic acid (NA $30 \mu \mathrm{g}$ ); metronidazole (MZ $5 \mu \mathrm{g}$ ); 2,3,5-triphenyltetrazolium chloride (TTC $1000 \mu \mathrm{g}$ ); 5-fluorouracil (FUR $80 \mu \mathrm{g}$ ) and sodium arsenite (SAR $30 \mu \mathrm{g}$ ).

A suspension of the test organism $(24 \mathrm{~h}$ growth at $37^{\circ} \mathrm{C}$ on biotyping agar) was prepared to a density equivalent to McFarland's Standard No. 2 in maximum recovery diluent (MRD) and then further diluted 1 in 100 in MRD. Biotyping agar plates were inoculated by flooding the surface with $1.5 \mathrm{ml}$ of the diluted bacterial suspension. Each plate was drained of excess liquid and dried at room temperature. The rings were placed on the surface of each plate, which was incubated under micro-aerobic conditions at $37^{\circ} \mathrm{C}$ for $24 \mathrm{~h}$. The inhibition zones were measured with calipers and results were scored according to the manufacturer's instructions. A proportion of cultures

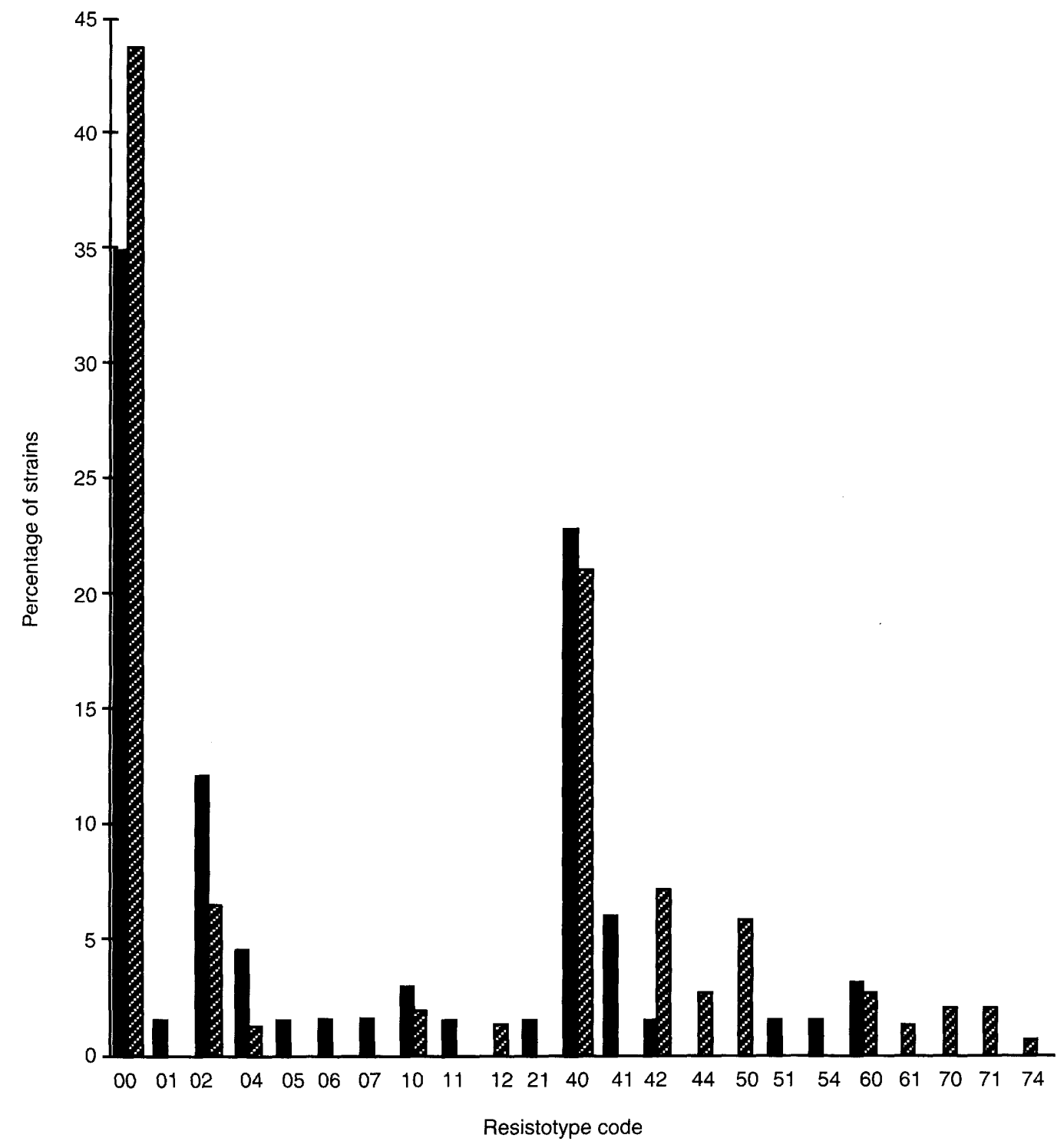

Fig. 1. Distribution of isolates according to resistotype at $24 \mathrm{~h}$ :

C. coli, C. jejuni. 
were then re-incubated for a further $24 \mathrm{~h}$ and zones were measured again.

\section{Results}

\section{Resistotypes at $24 \mathrm{~h}$}

A total of 14 resistotypes was recorded at $24 \mathrm{~h}$ amongst the 158 strains of $C$. jejuni and 16 resistotypes amongst the $70 \mathrm{C}$. coli strains tested. The distribution of strains according to resistotypes is shown in Fig. 1. There were two predominant types for $C$. jejuni with codes 00 $(44 \%)$ and $40(21 \%)$, and three for $C$. coli with codes $00(33 \%), 40(21 \%)$ and $02(11 \%)$. Code 00 strains were fully sensitive to all six agents whereas the isolates with code 40 were resistant to metronidazole and isolates with code 02 were resistant to 5fluorouracil. Strains with other codes were rarer and each accounted for $<7 \%$ of strains. Five strains $(2 \%)$ were non-typable as they did not grow within $24 \mathrm{~h}$ on Campylobacter biotyping agar when the inoculum was prepared according to manufacturer's instructions. When a higher inoculum was used ( 1 in 50 dilution of a bacterial suspension equivalent to McFarlands No. 2 ), results were obtained at $24 \mathrm{~h}$ for all four strains tested.

Two outbreak sets of $C$. jejuni strains were included in the study. Two of the four isolates from a waterassociated outbreak (HS1) were code 00 and two were code 02, whereas the five strains from a milkassociated outbreak (HS2) were all code 00 .

\section{Resistotypes at $48 \mathrm{~h}$}

Results for all $70 \mathrm{C}$. coli strains were recorded at $48 \mathrm{~h}$. Results for $42 C$. jejuni isolates were also re-read at $48 \mathrm{~h}$ when 24 -h results were equivocal because growth was either very light or just detectable within the zones causing them to be hazy and difficult to interpret. Sixty percent (42 of 70) of C. coli strains and 79\% (33 of 42) of the $C$. jejuni examined had a different resistotype at $48 \mathrm{~h}$ from that recorded at $24 \mathrm{~h}$. Results for the strains with changed resistotypes at $48 \mathrm{~h}$ are summarised in Table 1. Three new resistotypes (codes 46, 52 and 63) were observed at $48 \mathrm{~h}$ with $C$. jejuni and seven new resistotypes (codes 12, 15, 43, 46, 53, 56 and 62) were observed at $48 \mathrm{~h}$ with $C$. coli, but three codes were lost with $C$. coli. For many strains with altered resistotypes (49 of $75 ; 65 \%$ ), the differences were attributed to changes in the FUR results.

\section{Reproducibility of resistotypes at $24 \mathrm{~h}$}

The reproducibility of resistotyping results was investigated with a subset of 77 strains (41 from 158 of C. jejuni and 36 from 70 of C. coli). These strains were selected proportionally to represent the different resistotype codes encountered in the study. A total of $21(51 \%)$ of 41 strains of C. jejuni and $31(36 \%)$ of 36
C. coli strains had different initial and repeat codes as listed in Table 2. On repeat testing, four new codes (41, 46, 54 and 62) were gained and one was lost (74) for $C$. jejuni, and three new codes were gained $(12,15$ and $43)$ and two were lost (06 and 11) for $C$. coli. The changes in resistotype seen in $C$. jejuni were often associated with FUR $(52 \% ; 11$ of 21 strains) whereas

Table 1. Summary of results for strains of C. jejuni and $C$. coli with differing resistotypes at $24 \mathrm{~h}$ and at $48 \mathrm{~h}$

\begin{tabular}{|c|c|c|c|c|}
\hline \multicolumn{2}{|c|}{ Code } & \multicolumn{2}{|c|}{ Number of strains } & \multirow{2}{*}{$\begin{array}{l}\text { Agent associated } \\
\text { with } \\
\text { changed code }\end{array}$} \\
\hline $24 \mathrm{~h}$ & 48 & C. jejuni & C. coli & \\
\hline 00 & 01 & & 1 & TTC \\
\hline 00 & 02 & 19 & 14 & FUR \\
\hline 00 & 04 & 1 & & SAR \\
\hline 00 & 40 & 2 & & $\mathrm{MZ}$ \\
\hline 00 & 42 & 1 & & $\mathrm{FUR}+\mathrm{MZ}$ \\
\hline 00 & 46 & 2 & 2 & $\mathrm{MZ}+\mathrm{FUR}+\mathrm{SAR}$ \\
\hline 02 & 07 & & 5 & $\mathrm{TTC}+\mathrm{SAR}$ \\
\hline 04 & 06 & & 2 & FUR \\
\hline 06 & 07 & & 1 & TTC \\
\hline 10 & 12 & & 2 & FUR \\
\hline 11 & 15 & & 1 & SAR \\
\hline 40 & 41 & & 2 & TTC \\
\hline 40 & 42 & 2 & 3 & FUR \\
\hline 40 & 43 & & 4 & TTC + FUR \\
\hline 40 & 46 & 2 & & FUR \\
\hline 40 & 60 & 1 & & NA \\
\hline 41 & 43 & & 1 & FUR \\
\hline 41 & 51 & & 1 & $\mathrm{~T}$ \\
\hline 41 & 53 & & 1 & $\mathrm{~T}+$ FUR \\
\hline 42 & 44 & 1 & & $\mathrm{SAR}+\mathrm{FUR}$ \\
\hline 50 & 52 & 1 & & FUR \\
\hline 54 & 56 & & 1 & FUR \\
\hline 60 & 62 & & 1 & FUR \\
\hline 61 & 63 & 1 & & FUR \\
\hline
\end{tabular}

TTC, 2,3,5,triphenyltetrazolium chloride; NA, nalidixic acid; FUR 5-fluorouracil; T, tetracycline; SAR, sodium arsenite; MZ, metronidazole.

Table 2. Summary of results for strains of $C$. jejuni and C. coli with differing resistotypes on repetition

\begin{tabular}{|c|c|c|c|c|}
\hline \multicolumn{2}{|c|}{ Code } & \multicolumn{2}{|c|}{ Number of strains } & \multirow{2}{*}{$\begin{array}{l}\text { Agent associated } \\
\text { with } \\
\text { changed code* }\end{array}$} \\
\hline Initial & Repeat & C. jejuni & C. coli & \\
\hline 00 & 01 & & 2 & TTC \\
\hline 00 & 02 & 5 & & FUR \\
\hline 00 & 40 & 1 & & $\mathrm{MZ}$ \\
\hline 00 & 42 & 1 & & $\mathrm{FUR}+\mathrm{MZ}$ \\
\hline 02 & 00 & 1 & & FUR \\
\hline 02 & 04 & & 1 & SAR \\
\hline 02 & 07 & & 1 & $\mathrm{TTC}+\mathrm{SAR}$ \\
\hline 04 & 44 & 1 & & $\mathrm{MZ}$ \\
\hline 06 & 05 & & 1 & TTC + FUR \\
\hline 10 & 12 & & 1 & FUR \\
\hline 11 & 15 & & 1 & SAR \\
\hline 40 & 41 & & 4 & TTC \\
\hline 40 & 42 & 4 & & FUR \\
\hline 40 & 44 & 2 & & SAR \\
\hline 40 & 46 & 1 & & FUR + SAR \\
\hline 41 & 43 & & 1 & FUR \\
\hline 41 & 51 & & 1 & $\mathrm{~T}$ \\
\hline 60 & 62 & 1 & & FUR \\
\hline 70 & 71 & 1 & & TTC \\
\hline 71 & 40 & 1 & & $\mathrm{~T}+\mathrm{NA}+\mathrm{TTC}$ \\
\hline 71 & 41 & 1 & & $\mathrm{~T}+\mathrm{NA}$ \\
\hline 74 & 54 & 1 & & $\mathrm{NA}$ \\
\hline
\end{tabular}

*Agents as listed for Table 1. 
TTC-associated changes were most common in $C$. coli strains $(46 \% ; 6$ of 13$)$.

\section{Associations between resistotype and serotype}

Table 3 shows the distribution of $C$. jejuni and $C$. coli strains with respect to resistotypes (24-h result) and the main Penner serotypes studied (C. jejuni: HS1, HS2 and HS4 complex; C. coli: HS54, HS53, HS49 and HS46). Strains within each of the resistotypes were diverse with respect to species and serotype, with only four resistotypes and serotypes (10 strains) that were uniquely associated. Seven resistotypes were common to both species $(00,02,04,10,40,42$ and $60)$.

\section{Associations between resistotype and genotype}

Strains of C. jejuni and C. coli within each resistotype (24-h result) were compared with previously published genotypic data [6-8]. All resistotype groups, with the exception of that coded 12 (two $C$. jejuni strains) were diverse with respect to PFGE type. Overall, PFGE based on SmaI digests of genomic DNA was the most discriminatory method; for example, for $C$. jejuni, 21 different profiles were detected amongst the 70 strains of resistotype code 00 , and 17 amongst the 32 strains of code 40 . However, the four isolates from a waterassociated outbreak had identical PFGE and ribotypes, yet two of the strains were coded 00 and two were coded 02 . There were 28 different PFGE profiles in the C. coli strain set with six profiles amongst the 23 strains of resistotype code 00 , and nine different profiles amongst the 15 strains of code 40 . Table 3

Table 3. The coincidence of resistotypes within clonal lines* in C. jejuni and C. coli at $24 \mathrm{~h}$

\begin{tabular}{|c|c|c|}
\hline Clone identity ${ }^{\dagger}$ & $\begin{array}{c}\text { Number of } \\
\text { strains }\end{array}$ & $\begin{array}{l}\text { Mast resistotype } \\
\text { (number of strains) }\end{array}$ \\
\hline \multicolumn{3}{|l|}{ C. jejuni } \\
\hline HSI clonal line 1 & 10 & $00(6), 02(2), 40(2)$ \\
\hline HS 1 clonal line 2 & 4 & $00(1), 40(2), 42$ (1) \\
\hline HS1 clonal line 3 & 3 & $00(1), 40(2)$ \\
\hline HS1 clonal line 4 & 3 & $40(2), 61(1)$ \\
\hline HS2 clonal line 1 & 7 & $00(6), 40(1)$ \\
\hline HS2 clonal line 2 & 4 & $00(2), 02(1), 40(1)$ \\
\hline HS2 clonal line 3 & 4 & $42(2)$ \\
\hline HS4 clonal line 1 & 6 & $00(4), 02(1), 10(1)$ \\
\hline HS4 clonal line 2 & 6 & $\begin{array}{l}00(1), 02(1), 40(3), 42 \\
(1)\end{array}$ \\
\hline HS4 clonal line 3 & 5 & $00(5)$ \\
\hline HS4 clonal line 4 & 4 & $40(1), 44(3)$ \\
\hline HS4 clonal line 5 & 4 & $40(1), 50(3)$ \\
\hline HS4 clonal line 6 & 3 & $00(3)$ \\
\hline \multicolumn{3}{|l|}{ C. coli } \\
\hline HS54 clonal line & 13 & $00(10), 02(2), 10(1)$ \\
\hline HS53 clonal line & 7 & $00(7)$ \\
\hline HS49 clonal line & 7 & $02(5), 06(1), 07(1)$ \\
\hline HS46 clonal line 1 & 4 & $40(4)$ \\
\hline HS46 clonal line 2 & 4 & $00(4)$ \\
\hline
\end{tabular}

${ }^{*}$ Clonal lines defined on the basis of identity in serotype, ribotype, PFGE DNA profiles, and with the exception of C. jejuni HS2 strains, flagellin gene type [6-9].

$\dagger \mathrm{HS}$, serotype (heat stable antigen). lists the resistotypes recorded at $24 \mathrm{~h}$ that were associated with each of the clonal lines described previously. These clones were defined on the basis of identity in serotype, ribotype, PFGE DNA macrorestriction profiles and, in some cases, flagellin gene type. Most clones were not homogeneous at $24 \mathrm{~h}$ with respect to resistotype.

\section{Discussion}

Since 1984, biotypes within C. jejuni and C. coli have been identified mainly be the Lior scheme [3] and by the more discriminatory Preston scheme $[4,5]$. However, there are only limited data on the frequency and distribution of the biotypes in the UK [10] and the usefulness of biotyping for epidemiological purposes has not been clearly established. The Mast Campylobacter Biotyping Scheme is the first commercially available biotyping scheme for these organisms. The present results show that is was simple and fast to perform and gave a result for most strains within $24 \mathrm{~h}$. Compared with Lior biotyping, it provided improved discrimination between strains and typability was high at $24 \mathrm{~h}$. However, detailed analysis of the resistotyping results raised several important points relating to their interpretation.

First, the susceptibilities of some strains were difficult to score at $24 \mathrm{~h}$ and when this was due to poor growth or hazy zones there was a logical inclination to reincubate the plates and to score results at $48 \mathrm{~h}$. The study showed that the 48-h results provided no advantage in reproducibility or discrimination. In fact, it led to a shift in the spectrum of resistotypes, and a decrease in the overall level of discrimination for $C$. coli with code 02 becoming the single predominant type. From our strain set, it was clear that the FUR test was the most problematic of the agents and needs to be investigated with a larger number of both $C$. coli and $C$. jejuni strains of different serotypes. Poor growth at $24 \mathrm{~h}$ could be avoided, possibly by increasing the inoculum density, although the effect that might have on resistotyping results needs to be examined systematically. Thus culture conditions, such as the degree of dryness of biotyping agar plates, require careful monitoring because they may have a significant effect on the rate of growth.

Second, a lack of reproducibility was found in some instances when 24-h resistotyping results were repeated by the standard procedures. For $47 \%$ (16 of 34) of the retested strains the different code obtained on a single repetition was linked to the FUR test. The TTC results for $C$. coli also lacked reproducibility and were problematic for about one-third of strains. The results indicate that more extensive reproducibility studies are necessary to establish the reliability of the resistotyping scheme both within and between laboratories because of the difficulties of interpreting hazy zones. 
Degree of discrimination is a key criterion for assessing the value of a typing scheme [11]. The overall degree of discrimination achieved with resistotyping was clearly better than that of other biotyping schemes, with 14 different resistotypes of $C$. jejuni and 16 of $C$. coli in 228 strains compared with just four and two, respectively, with the Lior scheme. Furthermore, comparison with other typing methods indicated that resistotyping can discriminate within serotypes and ribotypes. However, a feature of the data was the fact that the majority $(65 \%)$ of the $C$. coli isolates belonged to just three resistotypes $(00,40$ and 02 ) and $65 \%$ of strains representing the most common UK serotypes of $C$. jejuni belonged to two resistotypes (codes 00 and 40). Further $C$. jejuni isolates representative of other serotypes need to be tested to establish if relative homogeneity is a consistent feature of the typing scheme rather than a result of biased strain selection. Every effort was made in the present study to randomise the strains used as much as possible with respect to host, time and location of isolation. Only four other resistotypes $(04,10,42$ and 60$)$ were common to both species, so there is a possibility that a proportion of resistotypes may be species-specific but more strains of $C$. jejuni need to be tested.

A desirable attribute of any typing scheme is that the types defined can be validated as far as possible by reference to other typing methods available for that micro-organism. Comparison of resistotyping data with Penner serotyping, an internationally recognised scheme, indicated considerable antigenic diversity within the predominant resistotypes. Similarly, comparison with genotypic data from ribotyping and PFGE profiling [6-8] also demonstrated a high level of intra-resistotype diversity. In contrast, four strains from a single outbreak which were identical with respect to serotype, PFGE profile and ribotype were divided into two groups by the resistotyping scheme, one coded 00 and the other coded 02 . It should be noted that this difference in codes is based on the FUR result, which was shown to be the least reproducible test. Overall, the results showed that resistotyping was not validated by reference to the other three typing methods, and that resistotyping did not provide a logical and valid basis for defining meaningful subtypes within $C$. jejuni and $C$. coli. In practice, the Mast system can be applied to screen for strains that do not share the same resistotypes. Where there are reproducible differences, it provides a marker for isolates that may be epidemiologically unrelated. However, strains with the same resistotype need to be investigated further by serotyping or by molecular typing to establish their affinities with more precision. For example, code 00 occurred in both outbreak sets of $C$. jejuni studied here yet the strains had different serotypes (HS1 and HS2) and could be further discriminated by PFGE profiling.

In conclusion, Mast resistotyping provides a simple and rapid approach to distinguishing between isolates within $C$. coli and strains of $C$. jejuni HS1, HS2 and the HS4 complex. However, as the system also groups apparently unrelated organisms, strains with the same resistotype must be subjected to further analysis by high resolution typing methods if the scheme is to be of value in future outbreak investigations. Factors that determine reproducibility of the test results need further evaluation, so that data from different laboratories can be compared with confidence.

The work was supported by a grant from the Department of Health, London.

\section{References}

1. Healing TD, Greenwood MH, Pearson AD. Campylobacters and enteritis. Rev Med Microbiol 1992; 3: 159-167.

2. Cowden JM, Wall PG, Adak G, Evans H, Le Baigue S, Ross D. Outbreaks of foodborne infectious intestinal disease in England and Wales: 1992 and 1993. Commun Dis Rep CDR Rev 1995; 5: R109-R117.

3. Lior H. New extended biotyping scheme for Campylobacter jejuni, Campylobacter coli, and "Campylobacter laridis". J Clin Microbiol 1984; 20: 636-640.

4. Bolton FJ, Holt AV, Hutchinson DN. Campylobacter biotyping scheme of epidemiological value. $J$ Clin Pathol 1984; 37: $677-681$

5. Bolton FJ, Wareing DRA, Skirrow MB, Hutchinson DN Identification and biotyping of campylobacters. In: Board RG, Jones D, Skinner FA (eds) Identification methods in applied and environmental microbiology. Society for Applied Bacteriology Technical Series 29. Oxford, Blackwell Scientific. 1992: $151-161$.

6. Owen RJ, Sutherland K, Fitzgerald C, Gibson J, Borman P, Stanley J. Molecular subtyping scheme for serotypes HSl and HS4 of Campylobacter jejuni. J Clin Microbiol 1995; 33: 872-877.

7. Gibson JR, Fitzgerald C, Owen RJ. Comparison of PFGE, ribotyping and phage-typing in the epidemiological analysis of Campylobacter jejuni serotype HS2 infections. Epidemiol Infect 1995; 115: 215-225.

8. Stanley J, Linton D, Sutherland K, Jones C, Owen RJ. Highresolution genotyping of Campylobacter coli identifies clones of epidemiologic and evolutionary significance. $J$ Infect Dis 1995; 172: 1130-1134.

9. Santesteban E, Gibson J, Owen RJ. Flagellin gene profiling of Campylobacter jejuni heat stable serotype 1 and 4 complex. Res Microbiol 1996 (in press).

10. Fricker CR, Park RWA. A two-year study of the distribution of 'thermophilic' campylobacters in human, environmental and food samples from the Reading area with particular reference to toxin production and heat-stable serotype. $J$ Appl Bacteriol 1989; 66: 477-490.

11. Hunter PR, Gaston MA. Numerical index of the discriminatory ability of typing systems: an application of Simpson's index of diversity. $J$ Clin Microbiol 1988; 26: 2465-2466. 